\title{
TURBULENCE WITHOUT PRESSURE: EXISTENCE OF THE INVARIANT MEASURE *
}

\author{
HENRY P. MCKEAN ${ }^{\dagger}$
}

1. Introduction. A number of proofs have been offered of the fact that Burgers' equation, with Brownian external force, settles down, with time, into a statistically steady state: see, for instance, Sinai [1996], E-Khanin-Mazel-Sinai [2000], and KuksinShirikyan [2001]. I propose a simple proof based on ideas of Döblin [1940] and Feller [1966]. The equation in question:

$$
\frac{\partial v}{\partial t}+v \frac{\partial v}{\partial x}=\frac{1}{2} \frac{\partial^{2} v}{\partial x^{2}}+e \frac{d b}{d t}
$$

represents an $\infty$-dimensional diffusion in the space of functions $v(x): 0 \leq x<1$ of period 1 say, with mean value $\int_{0}^{1} v=0$. The external force $e d b / d t$ is a sum of "modes" $e_{n}(x) \equiv$ a constant $e_{n} \times \sqrt{2} \sin / \cos (2 \pi n x)$, indexed by $n \geq 1$, multiplied each by the differential of its private 1-dimensional standard Brownian motion $b_{n}(t): 0 \leq t<\infty$. It is assumed for the present proof that all modes are active, i.e. $e_{n} \neq 0$ for any $n \geq 1$, and that the force is smooth in respect to $0 \leq x<1$, i.e. that $e_{n}$ vanishes rapidly; the second proviso permits you to realize the diffusion in the space $C^{\infty}[0,1)$. The force competes with the restoring drift $(1 / 2) \partial^{2} v / \partial x^{2}$, pulling back towards the origin as per $\int_{0}^{1} v v^{\prime \prime}=\int_{0}^{1}\left(v^{\prime}\right)^{2} \leq 0$, and with the twist $v \partial v / \partial x$, so-called because $\int_{0}^{1} v\left(v v^{\prime}\right)=0$, the outcome being the statistical steady state cited at the start. The simplicity of the present method has its price: in particular, it does not yield the exponentially fast convergence of $F_{t}(v) \equiv E_{v}\left[F\left(v_{t}\right)\right]$ to the invariant mean $\int F(v) d M(v)$, which must be a consequence of the rapid return of the diffusion to the vicinity of $v \equiv 0$. Observe, in this connection,

$$
\begin{gathered}
d \int_{0}^{1} v^{2}=-\int_{0}^{1}\left(v^{\prime}\right)^{2} d t+2 \int_{0}^{1} e v d b+\int_{0}^{1} e^{2} d t \\
\quad \leq-4 \pi^{2} \int_{0}^{1} v^{2} d t+2 \int_{0}^{1} e v d b+\int_{0}^{1} e^{2} d t
\end{gathered}
$$

with the obvious result that, up to the passage time $T=\min \left(t: \int_{0}^{1} v^{2}=r^{2}\right)$,

$$
e^{4 \pi^{2} t} r^{2} \leq e^{4 \pi^{2} t} \int_{0}^{1} v^{2} \leq \int_{0}^{1} v_{0}^{2}+2 \int_{0}^{t} e^{4 \pi^{2} s} \int_{0}^{1} e v d b+\int_{0}^{1} e^{2} \frac{e^{4 \pi^{2} t}-1}{4 \pi^{2}},
$$

which yields

$$
E_{v}\left(e^{4 \pi^{2} T}\right) \leq \frac{R^{2}}{r^{2}-\left(1 / 4 \pi^{2}\right) \int_{0}^{1} e^{2}} \text { for } R^{2}=\int_{0}^{1} v^{2}>r^{2}>\frac{1}{4 \pi^{2}} \int_{0}^{1} e^{2} .
$$

*Received July 2, 2002; accepted for publication December 13, 2002.

†CIMS, 251 Mercer Street, New York, NY 10012, USA (mckean@cims.nyu.edu). 
2. The Diffusion. The equation can be solved with the help of the Cole-Hopf substitution: if $w=\exp \left[-\int_{0}^{1} d \xi \int_{\xi}^{x} v(\eta) d \eta\right]$, then

$$
\frac{\partial w}{\partial t}=\frac{1}{2} \frac{\partial^{2} w}{\partial x^{2}}+w f d b+\frac{w}{2}\left[f^{2}-\int_{0}^{1}\left(\frac{w^{\prime}}{w}\right)^{2}\right]
$$

with $-f^{\prime}=e, w>0$ and $\int_{0}^{1} \ell n w=0$, and this equation yields to the FeynmanKaç formula: $w(t, x)=Z^{-1} E_{x}\left[\mathfrak{z} w\left(0, x_{t}\right)\right]$, in which $x(t): t \geq 0$ is an auxiliary 1dimensional standard Brownian motion,

$$
\mathfrak{z}=\exp \left[\int_{0}^{t} f\left(x_{t-s}\right) d b_{s}\right], \text { and } Z=\exp \left[\int_{0}^{1} \ln E \cdot(\mathfrak{z} w)\right]
$$

is a normalizer to keep $\int_{0}^{1} \ell n w \equiv 0$. The recipe may be re-expressed in terms of the auxiliary Brownian motion tied at $x(0)=0$ and $x(t)=0$. Then a simple application of Kolmogorov-Centsov shows that the path $w$ (and so also $v$ ) can be realized in the space of functions jointly of class $C[0, \infty)$ in respect to $t \geq 0$ and of class $C^{\infty}[0,1)$ in respect to $0 \leq x<1$. In this way the diffusion is constructed: $v=-w^{\prime} / w$. The aim is now to prove the existence of the limit $F_{\infty}(v) \lim _{t \uparrow \infty} E_{v}\left[F / v_{t}\right]$ and to identify it as the invariant mean $\int F(v) d M(v)$. Naturally, it is essential that the mass of the distribution of $v$ not run out to $\infty$. I dispose of this at once by the estimate employed at the end of Section 1 which yields

$$
E\left(\int_{0}^{1} v^{2}\right) \leq e^{-4 \pi^{2} t} \int_{0}^{1} v_{0}^{2}+\int_{0}^{1} e^{2} \frac{1}{4 \pi^{2}}\left(1-e^{-4 \pi^{2} t}\right)
$$

whence

$$
P\left(\int_{0}^{1} v^{2}>R^{2}\right) \leq R^{-2}\left[e^{-4 \pi^{2} t} r^{2}+\frac{1}{4 \pi^{2}} \int_{0}^{1} e^{2}\right] \text { with } r^{2}=\int_{0}^{1} v_{0}^{2} .
$$

3. Equicontinuity. Let $\dot{v}(t, x)$ be the functional gradient $\partial v(t, x) / \partial v(0, y)$ for fixed $0 \leq y<1$. You have $\partial \dot{v} / \partial t=(1 / 2) \partial^{2} \dot{v} / \partial x^{2}-(\partial / \partial x)(v \dot{v})$ with $\dot{v}(0, x) d x=$ the unit mass at $x=y$, and this may be solved by a combination of Cameron-Martin and Feynman-Kaç: to wit,

$$
\dot{v}(t, x)=E_{x}\left[e^{-\int_{0}^{t} v\left(t-s, x_{s}\right) d x_{s}-\frac{1}{2} \int_{0}^{t} v^{2}\left(t-s, x_{s}\right) d s-\int_{0}^{t} v^{\prime}\left(t-s, x_{s}\right) d s}, x_{t}=y\right]^{1}
$$

which reduces to

$$
E_{y}\left[e^{\int_{0}^{t} v\left(s, x_{s}\right) d x_{s}-\frac{1}{2} \int_{0}^{t} v^{2}\left(s, x_{s}\right) d s}, x_{t}=x\right] \equiv E_{y}\left[\mathfrak{v}, x_{t}=x\right]
$$

upon reversal of the auxiliary Brownian path as per $x(s) \rightarrow x(t-s)(s \leq t)$. Now the chain rule in function space applied to $F_{t}(v)=E_{v}\left[F\left(v_{t}\right)\right]={ }^{2} B M\left[F\left(v_{t}\right)\right]$ with $F$ of class $C^{1}[C[0,1) \rightarrow \mathbb{R}]$ and $v+\vartheta \Delta v$ in place of $v$, plain, yields

$$
F_{t}(v+\Delta v)-F_{t}(v)=\int_{0}^{1} d \vartheta \int_{0}^{1} \Delta v(y) d y B M \int_{0}^{1} \operatorname{grad} F E_{y}\left[\mathfrak{v}, x_{t}=x\right] d x
$$

\footnotetext{
${ }^{1} E\left[I, x_{t}=y\right]$ is short for the density $(\partial / \partial y) E\left[I, x_{t} \leq y\right]$.

${ }^{2} B M$ is the Brownian mean over the individual motions $b_{n}: n \geq 1$.
} 
with $\operatorname{grad} F$ taken at $v_{t}$, so that

$$
\left|F_{t}(v+\Delta v)-F_{t}(v)\right| \leq|\operatorname{grad} F|_{\infty} \int_{0}^{1}|\Delta v| d y E_{y}(\mathfrak{v}) \leq|\operatorname{grad} F|_{\infty}|\Delta v|_{\infty}
$$

in view of $E(\mathfrak{v}) \leq 1$. This provides compactness, permitting you to choose $\alpha=\alpha_{1}>$ $\alpha_{2}>$ etc. $\downarrow 0$ so as to make $G_{\alpha}(v)=\alpha \int_{0}^{\infty} e^{-\alpha t} F_{t}(v) d t$ converge to a function $G_{0}(v)$ of class $C[C[0,1) \rightarrow \mathbb{R}]$, uniformly on compact figures such as $K=\left(v: \int_{0}^{1}\left(v^{\prime}\right)^{2} \leq R^{2}\right)$. I prefer this mode of convergence to the plain $\lim _{t \uparrow \infty} F_{t}(v)$ as it avoids a difficulty with the non-compactness of $C[0,1)$.

4. $G_{0}(v)$ is Constant in Respect to $v$. The point is that the diffusion comes close to the origin $v \equiv 0$ so that the path emanating from that place is typical; this is the idea of Döblin [1940]. Let a small number $r$ and a big number $R$ be fixed, let $K$ be the compact figure $\left(v:\left(\int_{0}^{1} e v\right)^{2} \leq r^{2} \& \int_{0}^{1}\left(v^{\prime}\right)^{2} \leq R^{2}\right)$, and let $T$ be the smaller of the passage time to $K$ and an adjustable integer $N=1,2,3$ etc. Then

$$
G_{\alpha}(v)=\alpha E_{v}\left[\int_{0}^{T} e^{-\alpha t} F_{t}(v) d t\right]+E_{v}\left[e^{-\alpha T} G_{\alpha}\left(v_{T}\right)\right]
$$

implies 1) $G_{0}(v)=E_{v}\left[G_{0}\left(v_{T}\right)\right]$ since $\left.T \leq N ; 2\right)$ the same with $T$ now equal to the passage time to $K$, by making $N \uparrow \infty$; and 3) $G_{0}(v)=G_{0}(0)$ by making $r \downarrow 0$ so that $K$ shrinks to the origin. It is here that the proviso $e_{n} \neq 0(n \geq 1)$ is used. Of course 2 ) is correct only if the passage time to $K$ is finite with probability one. This is so provided $R$ is big enough.

Proof. If, for some small $r$ and big $R$, the passage time $T$ is infinite, then for every $t \geq 0$, either $\left(\int_{0}^{1} e v\right)^{2}>r^{2}$ or $\int_{0}^{1}\left(v^{\prime}\right)^{2}>R^{2}$. Let $E$ be the set of times $s \leq t$ when $\left(\int_{0}^{1} e v\right)^{2}>r^{2}$ and $E^{\prime}$ its complement, on which you must have $\int_{0}^{1}\left(v^{\prime}\right)^{2}>R^{2}$. Two cases arise.

Case 1: $\int_{0}^{\infty} e^{4 \pi^{2} t}\left(\int_{0}^{1} e v\right)^{2} d t<\infty$. Then

$$
\begin{gathered}
d \int_{0}^{1} v^{2}=-\int_{0}^{1} v^{\prime 2} d t+2 \int_{0}^{1} e v d b+\int_{0}^{1} e^{2} d t \\
\leq-\frac{1}{2} \int_{0}^{1}\left(v^{\prime}\right)^{2} d t-2 \pi^{2} \int_{0}^{1} v^{2} d t+2 \int_{0}^{1} e v d b+\int_{0}^{1} e^{2} d t,
\end{gathered}
$$

and the resulting estimate

$$
e^{2 \pi^{2} t} \int_{0}^{1} v^{2} \leq \int_{0}^{1} v_{0}^{2}-\frac{1}{2} \int_{0}^{t} e^{2 \pi^{2} s} \int_{0}^{1}\left(v^{\prime}\right)^{2} d s+2 \int_{0}^{t} e^{2 \pi^{2} s} \int_{0}^{1} e v d b+\int_{0}^{1} e^{2} \times \frac{e^{2 \pi^{2} t}}{2 \pi^{2}}
$$


implies $^{3}$

$$
\int_{0}^{t} d s e^{2 \pi^{2} s} \int_{0}^{1}\left(v^{\prime}\right)^{2} \leq \int_{0}^{1} e^{2} \times e^{2 \pi^{2} t} \quad \text { for } t \uparrow \infty .
$$

But now

$$
\begin{aligned}
2 e^{2 \pi^{2} t} \int_{0}^{1} e^{2} & \geq \int_{0}^{t} e^{2 \pi^{2} s}\left(\int_{0}^{1} e v\right)^{2}+\int_{0}^{t} e^{2 \pi^{2} s} \int_{0}^{1}\left(v^{\prime}\right)^{2} \\
& \geq r^{2} \int_{E} e^{4 \pi^{2} s}+R^{2} \int_{E^{\prime}} e^{2 \pi^{2} s}
\end{aligned}
$$

cannot be balanced as $t \uparrow \infty$ if $R$ is too big in comparison to $\int_{0}^{1} e^{2}$, no matter how small the fixed number $r>0$ may be.

Case 2: $\int_{0}^{\infty} e^{4 \pi^{2} t}\left(\int_{0}^{1} e v\right)^{2} d t=\infty$. You have

$e^{2 \pi^{2} t} \int_{0}^{1} v^{2} \leq \int_{0}^{1} v_{0}^{2}-\frac{1}{2} \int_{0}^{t} e^{2 \pi^{2} s} \int_{0}^{1}\left(v^{\prime}\right)^{2} d s+2 \int_{0}^{t} e^{2 \pi^{2} s} \int_{0}^{1} e v d b+\int_{0}^{1} e^{2} \times \frac{e^{2 \pi^{2} t}}{2 \pi^{2}}$

as before, and an application of the law of the iterated logarithm to the Brownian integral produces the over-estimate of the right side by

$\int_{0}^{1} v_{0}^{2}-\frac{1}{2} \int_{0}^{t} e^{2 \pi^{2} s} \int_{0}^{1}\left(v^{\prime}\right)^{2}-2 \sqrt{\int_{0}^{t} e^{4 \pi^{2} s}\left(\int_{0}^{1} e v\right)^{2} \times \ln \ell n}(\operatorname{ditto})+\int_{0}^{1} e^{2} \times e^{2 \pi^{2} t}$,

valid i.o. as $t \uparrow \infty^{4}$, so that, i.o.,

$$
N \times \sqrt{\int_{0}^{t} e^{4 \pi^{2} s}\left(\int_{0}^{1} e v\right)^{2}}+\frac{1}{2} \int_{0}^{t} e^{2 \pi^{2} s} \int_{0}^{1}\left(v^{\prime}\right)^{2}+\leq \int_{0}^{1} v_{0}^{2}+\int_{0}^{1} e^{2} \times e^{2 \pi^{2} t}
$$

for any $N=1,2,3$ etc. you like, and

$$
N r \sqrt{\int_{E} e^{4 \pi^{2} s}}+\frac{R^{2}}{2} \int_{E^{\prime}} e^{2 \pi^{2} s} \leq 2 \int_{0}^{1} e^{2} \times e^{2 \pi^{2} t} \text { i.o. }
$$

But then $\int_{E^{\prime}} e^{2 \pi^{2} s}$ is small compared to $e^{2 \pi^{2} t}, R$ being large, so that

$$
\int_{E} e^{4 \pi^{2} s}=\frac{e^{4 \pi^{2} t}-1}{4 \pi^{2}}-\int_{E^{\prime}} e^{4 \pi^{2} s} \geq \frac{e^{4 \pi^{2} t}-1}{4 \pi^{2}}-e^{2 \pi^{2} t} \int_{E^{\prime}} e^{2 \pi^{2} s}
$$

is comparable to $\left(1 / 4 \pi^{2}\right) e^{4 \pi^{2} t}$, and the preceding display may be unbalanced by choice of $N$.

\footnotetext{
${ }^{3} \int_{0}^{\infty} I d b$ is finite if $\int_{0}^{\infty} I^{2} d t<\infty$ for any non-anticipating $I$.

${ }^{4}$ The point is that $\int_{0}^{t} I d b$ looks like a standard 1-dimensional Brownian motion run with the clock $\int_{0}^{t} I^{2}$
} 
5. Identification of $G_{0}(0)$. To complete the proof, it is necessary to know that $G_{0}(0)$ does not depend upon the mode of approach of $\alpha$ to $0^{+}$. Then $G_{0}(0)=$ $\int F(v) d M(v)$ with invariant $M$ : in fact, $G_{\alpha}$ formed with $F_{t}(v)=E_{v}\left[F\left(v_{t}\right)\right]$ in place of $F$ is nothing but $E_{v}\left[G_{\alpha}\left(v_{t}\right)\right]$ with the old $G_{\alpha}$ so that

$$
\int F_{t}(v) d M(v)=E_{v}\left[G_{0}\left(v_{t}\right)\right]=G_{0}(0)=\int F(v) d M(v),
$$

as advertised. The uniqueness of the invariant measure is now self evident, too. The omitted identification of $G_{0}(0)$ is simple. Take $F \geq 0$ and let it vanish off the compact figure $K=\left(v: \int_{0}^{1}\left(v^{\prime}\right)^{2} \leq R^{2}\right)$. This is harmless to the generality of $F, R$ being adjustable. Let $m_{\alpha}$ be the maximum of $G_{\alpha}$; obviously, $m_{\alpha} \downarrow m_{0} \geq 0$ as $\alpha \downarrow 0$ and $G_{0} \leq m_{0}$. It is to be proved that $G_{0} \equiv m_{0}$.

Proof. Let $T$ be the passage time to $K$. Then, with the cut-off in $F, F\left(v_{t}\right)=0$ for $t \leq T$, and $G_{\alpha}(v)=E_{v}\left[e^{-\alpha T} G_{\alpha}\left(v_{T}\right)\right]$; in particular, $G_{\alpha}$ peaks at some place $v_{\alpha} \epsilon K$. Now, with $\alpha=$ the old $\alpha_{n}$ of $\S 3$ and $n \uparrow \infty$, you have $m_{\alpha}=G_{\alpha}\left(v_{\alpha}\right)$, and the convergence of $G_{\alpha}(v)$ to the constant $G_{0}(0)$, which is uniform on the compact $K$, implies $m_{0}=G_{0}\left(v_{0}\right)$ for some $v_{0} \epsilon K$. Then $m_{0}=G_{0}(0) —$ in short, the full $\lim _{\alpha \downarrow 0} G_{\alpha}(v)=m_{0}$ exists. This nice trick is adapted from Feller [1966].

\section{REFERENCES}

[1] DöBlin, W., Éléments d'une théorie générale des chaines constants simples de Markoff, Ann. Sci. École Norm. Sup., 631 (1940), pp. 317-356.

[2] E., W., E. K., Khanin, A., Mazel and Ya., Sinai, Invariant measures for Burgers equation with stochastic forcing, Ann. Math., 151 (2000), pp. 877-960.

[3] Feller, W., Introduction to Probability Theory and its Applications, John Wiley \& Sons, New York, 1966

[4] Kuksin, S., And A. Shirikyan, A coupling approach to randomly forced nonlinear PDEs, Comm. Math. Phys., 221 (2001), pp. 351-366.

[5] SinaI, YA., Burgers equation driven by a periodic stochastic flow, Itô's Stochastic Calculus and Probability Theory, pp 347-353. Springer-Verlag, New York, 1996. 
H. P. MCKEAN 